\section{Bone marrow stem cells are key regulators of cardiac repair following MI}

Bone marrow stem cell therapy following myocardial infarction (MI) has shown promise in recent clinical trials for cardiac regeneration, but the mechanisms of benefit are uncertain. To better understand the processes involved, Fazel et al. looked at endogenous myocardial repair involving the c-kit receptor-known to be expressed on bone marrow stem cells and putative cardiac stem cells - in a c-kit mutant $\mathrm{Kit} \mathrm{W}^{\mathrm{K}} \mathrm{Kit}{ }^{\mathrm{W}-\mathrm{v}}$ mouse model.

Wild-type control mice with induced MI had an increased number of $\mathrm{c}^{-\mathrm{kit}^{+}}$cells in their heart that were identified as being of bone marrow origin. These recruited bone marrow c-kit ${ }^{+}$cells were associated with increased proangiogenic cytokines, including vascular endothelial growth factor, around the infarct zone. Resulting endothelial mitogenesis in these areas was associated with the establishment of extensive myofibroblast-rich repair tissue. Mutations in the c-kit receptor in $\mathrm{Kit}^{\mathrm{W}} / \mathrm{Kit}{ }^{\mathrm{W}-\mathrm{v}}$ mice interfered with the mobilization of the c-kit ${ }^{+}$cells to the heart, prevented angiogenesis, reduced the formation of repair tissue, and led to cardiac failure and death. Irradiation followed by transplantation with wild-type bone marrow rescued the cardiomyopathic phenotype in $\mathrm{Kit}^{W} / \mathrm{Kit} \mathrm{W}^{\mathrm{W}-\mathrm{v}}$ mice.

The authors conclude that these results implicate bone marrow c-kit ${ }^{+}$cells as key regulators of the angiogenic switch seen in infarcted myocardium and, therefore, of efficient cardiac repair. These findings have implications for the use of allogenic bone marrow transplantation in $\mathrm{Ml}$, especially given that only temporary engraftment of the cells is needed.

Original article Fazel S et al. (2006) Cardioprotective c-kit ${ }^{+}$ cells are from the bone marrow and regulate the myocardial balance of angiogenic cytokines. J Clin Invest 116: 1865-1877

\section{Retinal vascular caliber analysis is useful in the prediction of CHD mortality}

A number of studies have indicated that microvascular disease might be a more important factor in the development of coronary heart disease $(\mathrm{CHD})$ in women than in men.
Photographs of the retinal microvasculature provide a noninvasive method for evaluating the condition of the microcirculation. Using this technique, the relationship between retinal vascular caliber and CHD-related death was examined in a population-based prospective study comprising 1,884 women and 1,456 men, aged 49 years or more, living in two postcode areas in New South Wales, Australia. In addition to stereoscopic retinal photographs, baseline measurements of height, weight, and blood pressure were taken for each participant. Preexisting CHD or diabetes was also recorded. Mean arteriolar and vascular caliber measurements were taken from baseline photographs, and arteriole-to-venule ratio calculated.

During the 9-year study, 192 participants (78 women and 114 men) died of CHD. Wider retinal venules were associated with an increased risk of CHD in both men and women aged 4975 years, even after adjustment for traditional risk factors. By contrast, smaller arteriole-tovenule ratios and narrower arteriolar vessels were strongly associated with a raised risk of death from CHD only in women aged 4975 years. No associations were found between $\mathrm{CHD}$ and retinal vascular caliber in participants $>75$ years old.

The researchers conclude that microvascular disease might have a role in CHD in middleaged individuals-especially women-and that the analysis of retinal microvasculature might provide a novel method for CHD risk assessment in individuals of both sexes under the age of 75 .

Original article Wang JJ et al. (2006) Retinal vascular calibre and the risk of coronary heart disease-related death. Heart [doi: 10.1136/hrt.2006.090522]

\section{Heterogeneous profile of LV remodeling in end-stage hypertrophic cardiomyopathy}

A large, multicenter study of patients with hypertrophic cardiomyopathy ( $\mathrm{HCM}$ ) has indicated that the end stage of the disease is less frequent and more heterogeneous in clinical profile than was previously thought. Harris et al. analyzed 1,259 patients with HCM enrolled at three centers in the US and Italy. End-stage $\mathrm{HCM}$ was found in 44 (3.5\%) patients; mean age at diagnosis was 45 years (range 1474 years). The mortality for end-stage HCM 